\title{
OP-0232 - Diabetes and risk of cancer incidence: results from a population-based cohort study in Northern Italy
}

Valeria Manicardi ${ }^{1}$, Paola Ballotari², Massimo Vicentini ${ }^{2}$, Marco Gallo3 , Sofia Chiatamone Ranieri ${ }^{5}$, Marina Greci6, Paolo Giorgi Rossi²

1 Department of Internal Medicine, Hospital of Montecchio, Local Health Authority of Reggio Emilia-IRCSS, Italy; ${ }^{2}$ Epidemiology Unit, Local Health Authority of Reggio Emilia-IRCSS, Italy; ${ }^{3}$ Oncological Endocrinology Unit, AOU Città della Salute e della Scienza di Torino, Italy; ${ }^{4} \mathrm{Clinical}$ Pathology and Microbiology Laboratory, Department of Laboratory Medicine, Local Health Authority of Teramo, Italy; ${ }^{5}$ Primary Health Care, Local Health Authority of Reggio Emilia-IRCSS, Italy

Background: There is considerable evidence linking diabetes and cancer incidence, with numerous epidemiological studies having identified associations between diabetes and several types of cancer, such as liver, pancreas, endometrium, kidney, breast, prostate, bladder, and colorectal cancer. There are several studies and systematic reviews comparing cancer incidence in groups of diabetic patients with incidence in the general population, but few have been able to study an entire population in which all subjects with diabetes were known. As the Reggio Emilia province has both a cancer and a diabetes registry, we were able to conduct a population-based cohort study.

Aims: The aim of this study was to compare cancer incidence between the population with and without diabetes, by cancer site. Further, we aimed to compare excess risk of cancer according to diabetes type and treatment.

Method: Using the Reggio Emilia diabetes registry (1) we classified the resident population aged 2084 at $31 / 12 / 2009$ as with and without diabetes. By linking this with the cancer registry we calculated the cancer incidence in 2010-2013 in the two populations. The incidence rate ratios (IRR) by cancer site, type of diabetes and treatment regimen were computed using the Poisson regression model.

Results: The cohort was composed by 383,799 subjects without diabetes and 23,358 with diabetes. During the follow-up period, we identified 1464 cancer cases among subjects with diabetes and 9858 in the remaining population. Prevalent diabetes at baseline was positively associated with total cancer incidence; the risk was slightly higher for females than for males (IRR=1.25, 95\%Cl 1.15-1.37 and $\mathrm{IRR}=1.22$, $95 \% \mathrm{Cl} 1.15-1.29$ respectively; test for heterogeneity $P=0.048$ ), with similar results focusing the analysis on subjects with at least 2 years of diabetes duration. Cancer sites driving the overall increased risk were liver $(\mathrm{IRR}=3.37,95 \% \mathrm{Cl} 2.63$ 4.32), pancreas (IRR=2.00, $95 \% \mathrm{Cl} 1.60-2.51)$, colon rectum (IRR=1.32, 95\% $\mathrm{Cl} 1.12-1.55)$, and bladder cancer $(\mathrm{IRR}=1.39,95 \% \mathrm{Cl} 1.16-1.68)$, corpus uteri (IRR=1.84, 95\%Cl 1.13-2.56).

There is also suggestion of an increased risk for kidney cancer in females and a decreased risk for prostate cancer. Excess risk was found in patients with type 2 diabetes, more pronounced among insulin users, especially with combined therapy (Tab.1).

\begin{tabular}{|c|c|c|c|c|}
\hline Tab.1 & $\begin{array}{l}\text { Person- } \\
\text { years }\end{array}$ & $\begin{array}{c}\mathbf{N} \\
\text { cancer }\end{array}$ & IRR & $95 \% \mathrm{Cl}$ \\
\hline Without diabetes & $1,499,890$ & 9858 & 1.00 & - \\
\hline With diabetes & 85,953 & 1464 & 1.22 & $1.15-1.29$ \\
\hline \multicolumn{5}{|l|}{ By type of diabetes: } \\
\hline Type 1 diabetes & 3017 & 15 & 0.88 & $0.53-1.47$ \\
\hline Secondary diabetes & 393 & 10 & 2.04 & $1.10-3.80$ \\
\hline Type 2 diabetes & 82,542 & 1439 & 1.22 & $1.15-1.29$ \\
\hline \multicolumn{5}{|c|}{ By treatment (only for Type 2 diabetes) } \\
\hline Diet only & 22,900 & 349 & 1.10 & $1.00-1.23$ \\
\hline OHAs only & 44,637 & 792 & 1.22 & $1.14-1.32$ \\
\hline Insulin only & 7738 & 161 & 1.32 & $1.13-1.54$ \\
\hline OHAs +insulin & 7267 & 137 & 1.37 & $1.16-1.62$ \\
\hline \multicolumn{5}{|c|}{ By diabetes duration (years): } \\
\hline $0-1$ & 24,553 & 361 & 1.10 & $0.99-1.23$ \\
\hline $2-5$ & 24,710 & 403 & 1.23 & $1.11-1.36$ \\
\hline $6-10$ & 17,033 & 337 & 1.44 & $1.29-1.61$ \\
\hline $11+$ & 19,658 & 363 & 1.15 & $1.04-1.30$ \\
\hline
\end{tabular}

Tab 1_Population, No. of cancer, Incidence Rate Ratios (IRR) and 95\% Confidence Intervals $(95 \% \mathrm{Cl})$ for type of diabetes, treatment (only for type 2 diabetes), and diabetes duration vs subjects without diabetes

Conclusion: The study suggests that there is an excess of cancer incidence in people with Type 2 diabetes, with differences among cancer sites. The strength of association depends on the specific cancer site, varies by gender and diabetes duration.

Insulin monotherapy and combined therapy per se, or as indicator of poor blood glucose control may play a role in the association between diabetes and some cancers. 